\title{
Combined lifestyle factors and risk of incident type 2 diabetes and prognosis among individuals with type 2 diabetes: a systematic review and meta-analysis of prospective cohort studies
}

\author{
Yanbo Zhang ${ }^{1,2} \cdot$ Xiong-Fei Pan ${ }^{1,2} \cdot$ Junxiang Chen ${ }^{1,2} \cdot$ Lu Xia $^{1,2} \cdot$ Anlan Cao $^{1,2} \cdot$ Yuge Zhang $^{1,2} \cdot$ Jing Wang ${ }^{3}$. \\ Huiqi $\mathrm{Li}^{1,2} \cdot$ Kun Yang ${ }^{4}$ Kunquan $\mathrm{Guo}^{4} \cdot$ Meian $\mathrm{He}^{2,5} \cdot$ An Pan $^{1,2}$
}

Received: 20 May 2019 / Accepted: 19 July 2019 / Published online: 4 September 2019

(C) Springer-Verlag GmbH Germany, part of Springer Nature 2019

\begin{abstract}
Aims/hypothesis A healthy lifestyle has been widely recommended for the prevention and management of type 2 diabetes. However, no systematic review has summarised the relationship between combined lifestyle factors (including, but not limited to, smoking, alcohol drinking, physical activity, diet and being overweight or obese) and incident type 2 diabetes and risk of health outcomes among diabetic individuals.

Methods EMBASE and PubMed were searched up to April 2019 without language restrictions. References included in articles in relevant publications were also screened. Cohort studies investigating the combined associations of at least three lifestyle factors with incident type 2 diabetes and health outcomes among diabetic individuals were included. Reviewers were paired and independently screened studies, extracted data and evaluated study quality. Random-effects models were used to calculate summary HRs. Heterogeneity and publication bias tests were also conducted.

Results Compared with participants considered to have the least-healthy lifestyle, those with the healthiest lifestyle had a $75 \%$ lower risk of incident diabetes (HR 0.25 [95\% CI 0.18, 0.35]; 14 studies with approximately 1 million participants). The associations were largely consistent and significant among individuals from different socioeconomic backgrounds and baseline characteristics. Among individuals with type 2 diabetes ( 10 studies with 34,385 participants), the HRs ( $95 \%$ CIs) were 0.44 (0.33, $0.60)$ for all-cause death, $0.51(0.30,0.86)$ for cardiovascular death, $0.69(0.47,1.00)$ for cancer death and $0.48(0.37,0.63)$ for incident cardiovascular disease when comparing the healthiest lifestyle with the least-healthy lifestyle.

Conclusions/interpretation Adoption of a healthy lifestyle is associated with substantial risk reduction in type 2 diabetes and long-term adverse outcomes among diabetic individuals. Tackling multiple risk factors, instead of concentrating on one certain lifestyle factor, should be the cornerstone for reducing the global burden of type 2 diabetes.
\end{abstract}

Keywords Cardiovascular disease $\cdot$ Lifestyle $\cdot$ Meta-analysis $\cdot$ Mortality $\cdot$ Systematic review $\cdot$ Type 2 diabetes

Electronic supplementary material The online version of this article (https://doi.org/10.1007/s00125-019-04985-9) contains peer-reviewed but unedited supplementary material, which is available to authorised users.

\section{An Pan}

panan@hust.edu.cn

1 Department of Epidemiology and Biostatistics, School of Public Health, Tongji Medical College, Huazhong University of Science and Technology, 13 Hangkong Road, Wuhan, China

2 Key Laboratory of Environment and Health, Ministry of Education \& Ministry of Environmental Protection, State Key Laboratory of Environmental Health (Incubating), School of Public Health, Tongji Medical College, Huazhong University of Science and Technology, Wuhan, China
3 Department of Forensic Medicine, Tongji Medical College, Huazhong University of Science and Technology, Wuhan, China

4 Department of Endocrinology, Affiliated Dongfeng Hospital, Hubei University of Medicine, Shiyan, China

5 Department of Occupational and Environmental Health, School of Public Health, Tongji Medical College, Huazhong University of Science and Technology, Wuhan, China 


\section{Research in context}

\section{What is already known about this subject?}

- Individual lifestyle factors, such as smoking, alcohol drinking, physical activity, diet and being overweight/obese, are associated with incident type 2 diabetes and prognosis among individuals with type 2 diabetes

- An increasing number of cohort studies report that combinations of healthy lifestyle factors are associated with a substantially lower risk of incident type 2 diabetes and lower risks of death and incident cardiovascular disease (CVD) among diabetic individuals

- No systematic review or meta-analysis to date has summarised the evidence on this topic

\section{What is the key question?}

- To what extent is the risk reduction in type 2 diabetes and the risk reduction in mortality and CVD among diabetic individuals associated with adherence to a healthy lifestyle?

\section{What are the new findings?}

- Participants with the healthiest lifestyle group had a 75\% lower risk of incident type 2 diabetes compared with those with the least-healthy lifestyle

- This association was consistent among populations from different socioeconomic backgrounds and with different baseline characteristics

- Among diabetic individuals, adopting the healthiest lifestyle was associated with a $31-56 \%$ risk reduction for total and cause-specific mortality and with a $52 \%$ reduction in incident CVD

\section{How might this impact on clinical practice in the foreseeable future?}

- Each country or region should formulate policies according to the local socioeconomic environment and public health practice to facilitate healthy lifestyle modifications. Furthermore, healthcare providers should encourage individuals at high risk of diabetes and those with diabetes to tackle multiple risk factors for the prevention and management of type 2 diabetes

\section{Abbreviations \\ CVD Cardiovascular disease \\ IQR Interquartile range \\ LS7 Life's Simple 7 \\ NOS Newcastle-Ottawa Scale}

\section{Introduction}

As one of the four major non-communicable diseases, type 2 diabetes has become a major public health challenge in both developed and developing countries. The most recent Global Burden of Disease Study estimated that there were over half a billion individuals with type 2 diabetes in 2017 globally and each year 22 million new cases were documented [1]. Diabetes complications, particularly cardiovascular disease (CVD), are the leading cause of morbidity and mortality among individuals with type 2 diabetes $[2,3]$. Therefore, prevention of type 2 diabetes and its long-term adverse outcomes is urgently needed to meet the Sustainable Development Goal target [4].
Strong evidence indicates that adopting a healthy lifestyle (i.e. maintaining a healthy body weight, following a healthy diet, exercising daily for at least $30 \mathrm{~min}$, avoiding smoking and avoiding harmful alcohol drinking) is a 'best buy' intervention for prevention and management of type 2 diabetes [3, 5]. Several large randomised controlled trials have found that lifestyle intervention was effective for the prevention of type 2 diabetes [6-9]. However, these trials were conducted in individuals with impaired glucose tolerance or impaired fasting glucose and the interventions were restricted to increasing physical activity level, adhering to a healthy diet and maintaining a healthy body weight. In addition, compared with observational studies, the numbers of participants in these trials were small and the follow-up durations were short. Hence, evidence from large prospective observational studies is still needed to examine the relationship between combined lifestyle factors and incident type 2 diabetes and its long-term outcomes; this is essential for making health policies and establishing clinical guidelines. Accordingly, we conducted this systematic review and meta-analysis to thoroughly evaluate the relationship between combined lifestyle factors and incident type 2 diabetes, as well as mortality and morbidity 
outcomes in diabetic individuals. Stratified analyses were also conducted to examine whether the associations were consistent across different characteristics of the participants.

\section{Methods}

This systematic review was registered on PROSPERO (CRD42018109642) and conducted according to the Metaanalysis Of Observational Studies in Epidemiology guideline [10].

Data sources and searches PubMed and EMBASE were searched for studies investigating the relationship between combined lifestyle factors and incident type 2 diabetes, as well as the risk of total and cause-specific mortality, incident CVD or its subtypes and cancer or site-specific cancer in diabetic individuals from database inception to 26 April 2019 by YbZ and JC. The details of the search terms are shown in the electronic supplementary material (ESM) Tables 1, 2. In brief, the search terms included the Medical Subject Heading terms and related exploded versions as well as keywords in titles or abstracts related to the following themes: 'diabetes', 'cardiovascular disease', 'cancer', 'mortality', 'combined', 'lifestyle' and 'cohort studies'. The search themes were then combined using the Boolean operator 'or' for the four health outcomes (diabetes, CVD, cancer and mortality) and then combined with other themes using 'and'. No language restriction was applied. In addition, reference lists of the included studies and relevant reviews were searched to identify further publications.

Study selection Prospective cohort studies were included if the study reported the relations of combined lifestyle factors with pre-determined outcomes. The lifestyle factors included but were not limited to smoking, alcohol drinking, physical activity and/or sedentary behaviour, diet, being overweight and/or obese and sleep duration and/or quality. Some studies additionally included metabolic factors, such as blood pressure, blood glucose and blood lipid levels, in the Life's Simple 7 (LS7) score defined by the American Heart Association and were also included in our main analysis. There were two major score systems: simple score, giving equal weight to each behavioural factor (e.g. most studies assigned ' 1 ' or ' 0 ' to individuals with or without a certain behaviour) [11] and LS7 score $[12,13]$ (ESM Table 3). We did not restrict the characteristics of the participants in the main analysis and studies with samples from a specific occupational group were also included.

Studies were excluded if they met the following criteria: (1) the study was unrelated to the exposures or pre-defined outcomes; (2) the study was from a different publication type (such as protocol, review, cross-sectional study, case-control study or animal experiment) or was not from a peer-reviewed publication (such as meeting abstract, editorial or commentary); (3) the study focused on a single lifestyle factor or combinations of only two lifestyle factors (we assumed that two factors could not reflect the overall lifestyle); (4) the study had less than 1 year of follow-up; (5) the study was a formulation or validation of prediction models; (6) duplicate publications or duplicate reporting from the same cohort studies; (7) the study investigated the association between combined lifestyle factors and mortality, incident CVD or incident cancer in participants without diabetes and (8) the study did not have necessary or sufficient data. We did not include conference abstracts in our analysis, but for a conference abstract that reported the associations between combined lifestyle factors and certain outcomes of interest, we searched online and also contacted the authors to inquire whether the full text had been published in peer-reviewed journals or accepted but not published online yet. This procedure ensured that we did not miss any potential eligible studies.

$\mathrm{YbZ}$ screened all the citations and another group of investigators, including LX, AC, YgZ, JW, HL and JC, also independently performed the study selection. Divergences were resolved by consensus or by consulting with the senior investigator (AP). The consistency of study selection before fulltext reading between reviewers was $99.92 \%$ (62 divergences among 82,208 citations, mostly due to different understandings of the included lifestyle factors).

Data extraction and quality assessment $\mathrm{YbZ}$ extracted all data and evaluated the quality of literature independently. Another group of investigators, including LX, AC, YgZ, JW, HL and $\mathrm{JC}$, also independently performed data extraction and quality assessment. Divergences were resolved by consensus or by consulting with the senior investigator (AP).

The following information was extracted using standardised tables: title, first author, publication year, cohort name, country, study duration and mean/median follow-up duration, sample size, outcome definition and attainment, the definitions of the healthy lifestyle factors and the characteristics of the participants, including age (mean/median and range), sex composition, race and ethnicity, education level and health status. For articles with insufficient data or unclear information, the corresponding authors were contacted (at least two attempts were made).

The Newcastle-Ottawa Scale (NOS) was used to evaluate the study quality [14], which focused on the selection of the study groups (four scores), the comparability of the groups (two scores) and the ascertainment of outcome (three scores).

Data synthesis and analysis Meta-analyses were performed by Stata software (version 14.0; StataCorp, College Station, TX, USA). HR was commonly used as the effect size in the original studies and was thus used in the pooled estimate. RR was used in some studies and was considered to be 
interchangeable with HR. The OR was transformed into RR using the following formula: $\mathrm{RR}=\mathrm{OR} /\left[\left(1-\mathrm{P}_{0}\right)+\left(\mathrm{P}_{0} \times \mathrm{OR}\right)\right]$, where $\mathrm{P}_{0}$ is the risk of an event in the non-exposed group [15]. The healthy lifestyle scores were constructed in multiple ways (different numbers or combinations of lifestyle factors and different weights for certain lifestyle factors) in various studies but were generally re-classified into three, four or five groups based on the distribution of the score in the study population. We pooled the HRs comparing participants in the highest score group with those in the lowest score group to represent the risk estimate comparing the healthiest vs least-healthy lifestyle. Random-effects models were used for data syntheses to allow heterogeneity from different study populations and score systems among different studies and the weights were equal to the inverse variance of each study's effect estimation. Forest plots were used to visualise the effect sizes and 95\% CIs across studies.

Heterogeneity across studies was assessed by $I^{2}$ statistic (ranging from $0 \%$ to $100 \%$ ), with a small value indicating less heterogeneity [16]. Pre-specified stratified analyses were conducted according to the study characteristics (such as study location, mean/median follow-up duration and different combinations of lifestyle factors) and population characteristics (age group, sex, race and ethnicity and education level). The $p$ values for difference between subgroups were also tested using meta-regression [16].

Publication bias was assessed by Begg and Mazumdar rank correlation test, Egger's test and the fail-safe $N$ statistic. If significant publication bias was indicated, Duval and Tweedie's trim and fill method was used to generate the 'unbiased' estimates by adding hypothesised studies to make the funnel plot symmetrical [16].

\section{Results}

Study selection and characteristics Based on the search strategy, 82,208 unique citations were identified and 82,169 articles were excluded after screening for the titles and abstracts according to the inclusion/exclusion criteria. Through manual inspections of the full text, 13 studies were excluded (see ESM Table 4). Finally, 16 studies [11, 12, 17-30] (among which, two studies $[18,29]$ were only used for stratified analyses) with 1,116,248 participants were included for meta-analyses of incident type 2 diabetes and ten studies [31-40] with 34,385 diabetic individuals were included for meta-analyses of mortality and incident CVD. No study investigated the association between combined lifestyle factors and incident cancer among diabetic individuals. The detailed procedure is shown in Fig. 1.

The characteristics of the eligible studies on incident type 2 diabetes are shown in Table 1 and ESM Table 5. Among 14 studies used for the main analysis, six were from the USA, three from Asia, three from Europe and two from Oceania; 12 were from high-income countries. One study reported results for men and women separately [11] and 13 studies reported results in men and women together (among which, four studies $[23,24,26,30]$ also conducted stratified analyses according to sex). The mean baseline age ranged from 38.0 years to 72.7 years (median 50.7, interquartile range [IQR] 10.3 years). The sample size ranged from 1639 to 461,211 . The mean/median follow-up duration ranged from 2.7 years to 20.8 years and the median (IQR) was 7.8 (3.2) years. The NOS scores of these studies were all $\geq 5$ (ESM Table 6).

The characteristics of the eligible studies on mortality and CVD risk among individuals with type 2 diabetes are shown in Table 1 and ESM Table 7. Three studies were from the USA, two were from Asia and four were from Europe; all studies were conducted in high-income countries or regions. Besides, one study [32] was a global study across several continents. The mean baseline age ranged from 45.8 years to 69.0 years (median 61.9 years, IQR 5.6 years). The sample size ranged from 592 to 11,527 . The mean/median follow-up duration ranged from 4.0 years to 20.6 years. The NOS scores of these studies were all $\geq 7$ (ESM Table 6).

Association of combined lifestyle factors with incident type 2 diabetes Fourteen studies (970,170 participants and 45,969 cases) reported results comparing participants with the healthiest vs least-healthy lifestyles for incident type 2 diabetes and the pooled HR $(95 \% \mathrm{CI})$ was $0.25\left(0.18,0.35 ; I^{2}=95.9 \%\right.$; Fig. 2).

The associations remained in all stratified analyses and no between-group differences were found (Fig. 3). Begg and Mazumdar rank correlation test, Egger's test and the classic fail-safe $N$ statistics indicated a small possibility of publication bias (ESM Table 8 and ESM Fig. 1).

Associations of combined lifestyle factors with mortality risk and incident CVD among diabetic individuals Figure 4 shows the associations between combined lifestyle factors and mortality risk and incident CVD among diabetic individuals. Compared with individuals with the least-healthy lifestyle, those with the healthiest lifestyle had a $56 \%$ lower risk of all-cause mortality (HR 0.44 [95\% CI $0.33,0.60] ; I^{2}=$ $74.1 \%$; seven studies), $49 \%$ lower risk of CVD mortality (HR 0.51 [95\% CI $0.30,0.86] ; I^{2}=70.5 \%$; four studies), $31 \%$ lower risk of cancer mortality (HR 0.69 [95\% CI 0.47 , $1.00] ; I^{2}=0.0 \%$; three studies) and $52 \%$ lower risk of incident CVD (HR 0.48 [95\% CI 0.37, 0.63]; $I^{2}=0.0 \%$; three studies).

\section{Discussion}

In this systematic review and meta-analysis of prospective cohort studies, the combination of multiple healthy lifestyle 
Fig. 1 Flowchart of study selection. T2D, type 2 diabetes

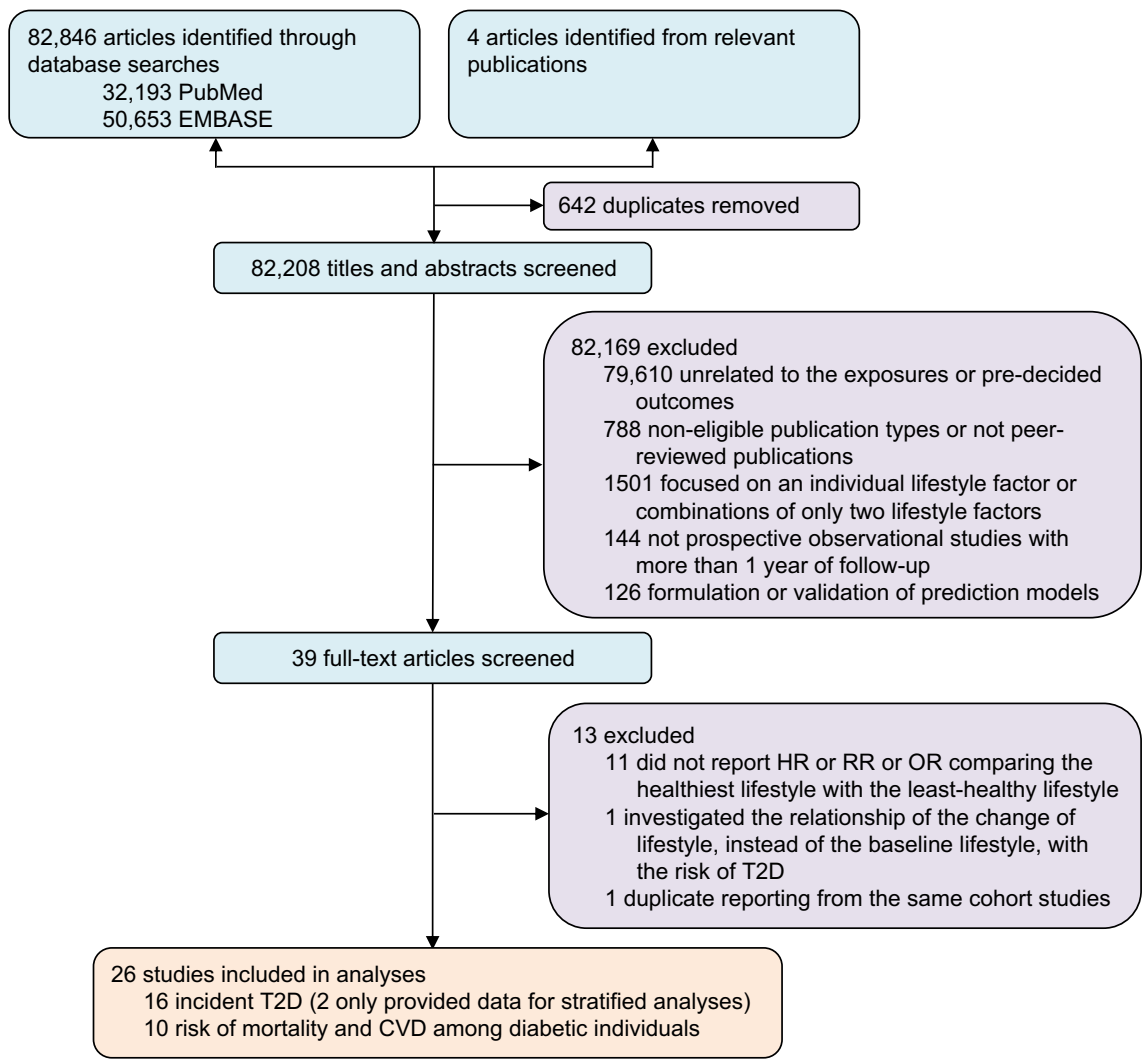

factors was associated with a substantially lower risk of incident type 2 diabetes. Compared with individuals with the least-healthy lifestyle, those with the healthiest lifestyle would have a $75 \%$ lower risk of incident type 2 diabetes. The associations were consistent among populations from different socioeconomic backgrounds and baseline characteristics. Moreover, adopting a healthy lifestyle was associated with a $56 \%, 49 \%, 31 \%$ and $52 \%$ lower risk of all-cause mortality, CVD mortality, cancer mortality and incident CVD among diabetic individuals.

To the best of our knowledge, our study is the first systematic review and meta-analysis investigating the association between combined lifestyle factors and incident type 2 diabetes. The result was consistent with those from several randomised controlled trials. The Da Qing Diabetes Prevention Outcome Study [41] recruited 577 Chinese adults with impaired glucose tolerance, among which 438 received dietary inventions, exercise interventions or both for 6 years. The participants who received lifestyle interventions had a $43 \%$ lower incidence of type 2 diabetes over 20 years. The Diabetes Prevention Program [8] in the USA enrolled 3234 overweight individuals with impaired glucose tolerance, of which 1079 received intensive lifestyle interventions through a healthy diet (low-energy, low-fat) and moderate physical activity aimed at reducing body weight by $7 \%$. After a mean of 2.8 years of follow-up, the lifestyle intervention group had a
$58 \%$ lower incidence of type 2 diabetes. The Finnish Diabetes Prevention Study [9] was conducted in 522 obese individuals with impaired glucose tolerance, of which 265 received 4 years of intensive lifestyle counselling for reducing body weight by $5 \%$ through a healthy diet (low-energy, low-saturated fat, high-fibre) and daily moderate physical activity. The participants who received the intervention displayed a $43 \%$ reduction in risk of type 2 diabetes. Incorporating those results with some other small randomised controlled trials, a meta-analysis found that lifestyle modification was associated with an RR $(95 \% \mathrm{CI})$ of $0.61(0.54,0.68)$ at the end of the active intervention [7]. However, these trials were conducted in relatively small samples from high-risk populations and the interventions only focused on diet, physical activity and body weight. This might explain why our results seemed stronger, indicating that longitudinal prospective cohort studies in the general population are essential for comprehensively understanding the association between lifestyle and incident type 2 diabetes.

Apart from overall lifestyle pattern, the association between an individual's healthy lifestyle factors and incident type 2 diabetes has been well established and adopted by the WHO and numerous authorities and organisations [42-44]. Previous studies found that a high level of physical activity was associated with a $35 \%$ lower risk of type 2 diabetes [45]. A healthy diet, no matter which diet score was adopted, was associated with 13-21\% lower risk of 


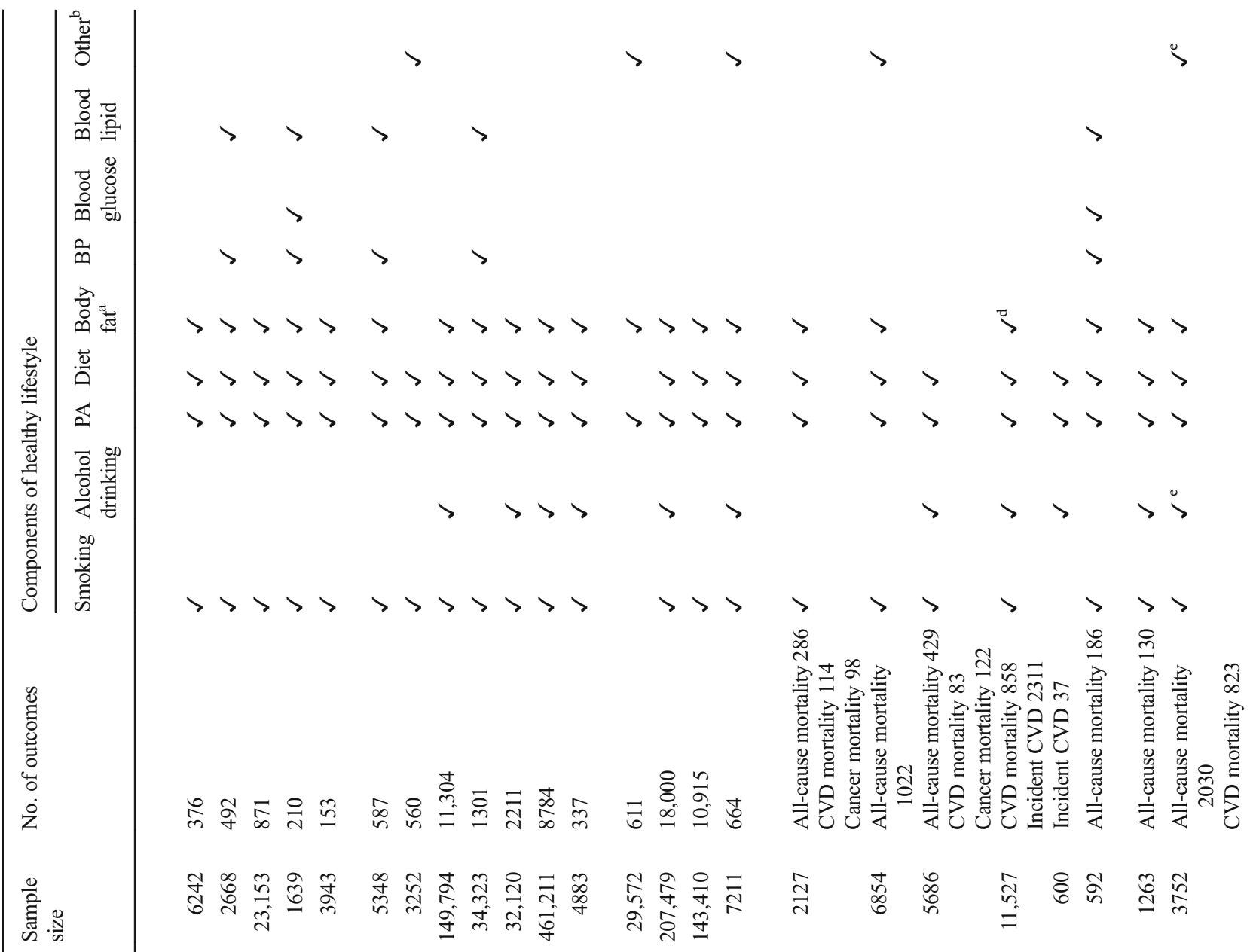

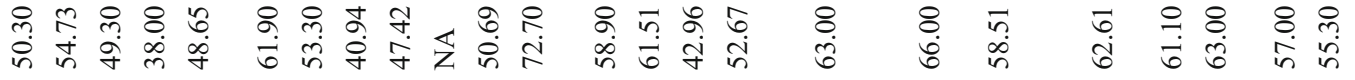

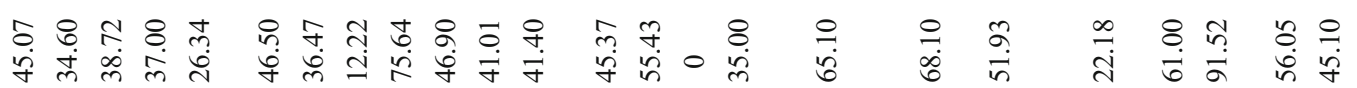

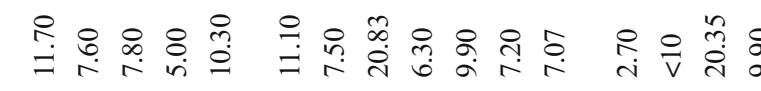




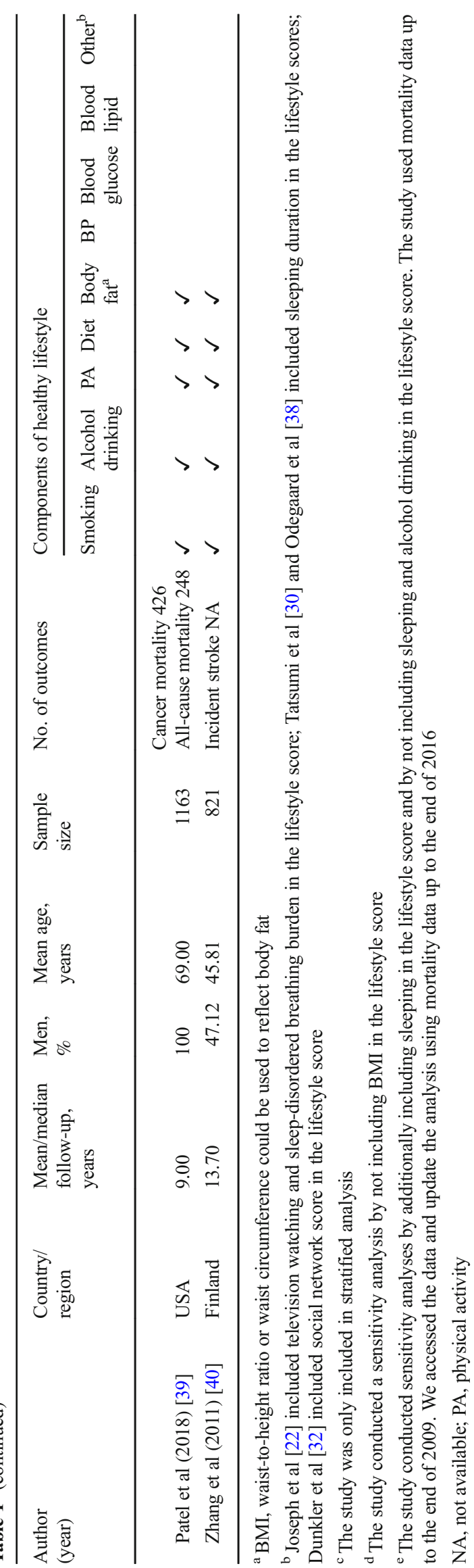




\begin{tabular}{|c|c|c|c|c|}
\hline \multirow{2}{*}{$\begin{array}{l}\text { Study } \\
\text { Dow et al (2019) [17] }\end{array}$} & \multirow{2}{*}{$\frac{\text { Comparison }}{3.5-4 \text { vs } 0-2 \text { points }}$} & \multicolumn{2}{|c|}{$\mathrm{HR}(95 \% \mathrm{Cl})$} & \multirow{2}{*}{$\begin{array}{r}\text { Weight, \% } \\
6.13\end{array}$} \\
\hline & & 一 & $0.30(0.17,0.52)$ & \\
\hline Ford et al (2009) [19] & 4 vs 0 points & $\mapsto$ & $0.07(0.05,0.12)$ & 6.54 \\
\hline Fretts et al (2014) [12] a & $4-7$ vs $0-1$ points & $\longmapsto$ & $0.14(0.07,0.26)$ & 5.57 \\
\hline Joosten et al (2010) (teetotallers) [20] b & $3-4$ vs $0-1$ points & $\longmapsto$ & $0.26(0.16,0.42)$ & 6.32 \\
\hline Joseph et al (2016) [21] & $4-6$ vs $0-1$ points & 1 & $0.25(0.18,0.35)$ & 6.88 \\
\hline Joseph et al (2017) [22] & $8-11$ vs $0-3$ points & . & $0.71(0.49,1.03)$ & 6.76 \\
\hline Li et al (2015) [23] ${ }^{\mathrm{C}}$ & $4-5$ vs $0-1$ points & ner & $0.14(0.12,0.16)$ & 7.30 \\
\hline Liu et al (2016) [24] a & Q5 vs Q1 & - 1 & $0.41(0.32,0.50)$ & 7.17 \\
\hline Long et al (2015) [25] & 6 vs $0-1$ points & & $0.27(0.18,0.40)$ & 6.66 \\
\hline Lv et al (2017) [26] ${ }^{d}$ & $4-6$ vs 0 points & 10 & $0.16(0.14,0.19)$ & 7.28 \\
\hline Mozaffarian et al (2009) [27] ${ }^{\mathrm{e}}$ & $3-4$ vs 0 points & - & $0.32(0.21,0.47)$ & 6.66 \\
\hline Nguyen et al (2017) [28] a & 6 vs 0 points & - & $0.18(0.10,0.32)$ & 6.12 \\
\hline Reis et al (2011) (men) [11] ${ }^{a}$ & 4 vs 0 points & - & $0.49(0.45,0.55)$ & 7.35 \\
\hline Reis et al (2011) (women) [11] a & 4 vs 0 points & :-1 & $0.35(0.27,0.44)$ & 7.12 \\
\hline Tatsumi et al (2013) [30] & 7 vs $1-2$ points & ら- & $0.31(0.18,0.53)$ & 6.16 \\
\hline Overall $(R=95.9 \%, p<0.001)$ & Highest vs lowest & $r+1$ & $0.25(0.18,0.35)$ & 100.00 \\
\hline
\end{tabular}

Fig. 2 Association of combined lifestyle factors with incident diabetes. The forest plot shows the HRs (circles) and 95\% CIs comparing people with the healthiest (highest score group) vs least-healthy (lowest score group) lifestyles for incident diabetes. The diamond represents the pooled HR. ${ }^{\text {a }}$ The ORs were reported in these studies and were transformed into RRs, which were then used in the pooled analysis. ${ }^{\mathrm{b}}$ The study included teetotallers and alcohol consumers; however, only the results for teetotallers were reported. ${ }^{\mathrm{c}}$ The data were provided by the authors. ${ }^{\mathrm{d}}$ There were

type 2 diabetes [46]. Besides, current smokers suffered a $37 \%$ higher risk of type 2 diabetes compared with never smokers [47]. It was also reported that moderate drinking (10-14 g alcohol per day) was associated with an $18 \%$ lower risk of type 2 diabetes compared with abstainers [48]. The strongest association was observed between body weight and incident type 2 diabetes: overweight and obese individuals displayed a $133 \%$ and $510 \%$ higher risk of type 2 diabetes, respectively, compared with their normalweight counterparts [49]. Our stratified analyses also showed that the HR was 0.37 when BMI was not included in the lifestyle score compared with 0.21 when it was included, although the comparison was not statistically significant. Although body weight plays a dominant role in the risk of type 2 diabetes, its individual association with incident type 2 diabetes was weaker than that of combined lifestyle factors. In addition, it is well-known that lifestyle behaviours, such as physical activity, diet quality and sleep pattern, are associated with body weight [50]. Besides, several studies reported that each additional healthy lifestyle factor was associated with 11-61\% lower risk of incident type 2 diabetes [11, 17, 18, 21, 23, 24, 27]. Hence, encouraging the population to adopt an overall healthy lifestyle is necessary for the prevention of type 2 diabetes.

The associations between combined lifestyle factors and incident type 2 diabetes were largely consistent across different age only 1243 participants and eight cases in the group with the highest score (individuals with 5 or 6 healthy lifestyle factors, i.e. points). Hence, we pooled this group with the second-highest score group (individuals with 4 points) using a fixed-effect model. ${ }^{\mathrm{e}}$ There were only 244 participants with unknown case numbers in the highest score group (individuals with 4 points). Hence, we pooled this group with the second-highest score group (individuals with 3 points) using a fixed-effect model

groups, sexes, geographical regions, economic levels, races and ethnicities and education levels, which may have important public health implications. People from different socioeconomic backgrounds may perceive and choose healthy lifestyles differently since socioeconomic factors are important determinants of lifestyle behaviours. For instance, individuals with higher education levels are less likely to smoke [51] and low-income populations consume more unhealthy foods because of low accessibility and high prices of healthy foods [52]. The number of diabetic individuals was large in non-high-income countries, whereas the majority of health expenditure for diabetes was in high-income countries [53, 54]. In addition, the implementation of health policies, such as tobacco control, avoidance of harmful use of alcohol and improvement of food quality, varied between different countries and regions [55]. Hence, each country or region should formulate policies tailored to the preference of local population or public health practice, in order to accelerate the progressions of meeting Sustainable Development Goal target 3.4 [4]. However, although most studies adjusted for some of these socioeconomic factors, few studies fully adjusted for them. Considering that socioeconomic factors could be upstream determinants of lifestyle, there might be some residual confounding not being adjusted for in the original studies.

Another public health issue is whether healthy lifestyles play an equally important role in preventing type 2 diabetes among high-risk populations and the general population. A 


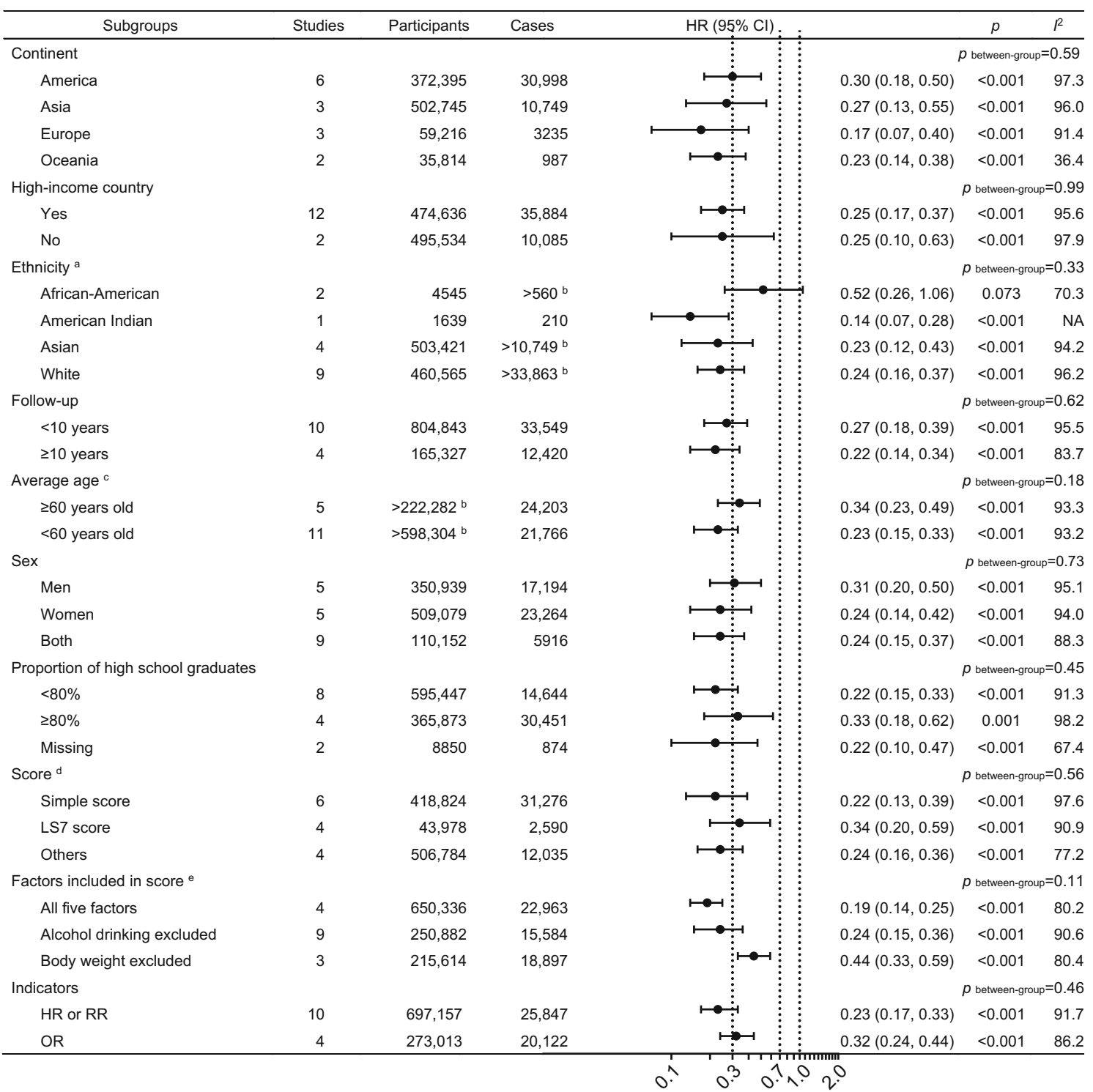

Fig. 3 Association of combined lifestyle factors with incident type 2 diabetes in different subgroups. The forest plot shows the HRs (circles) and $95 \%$ CIs comparing people with the healthiest (highest score group)

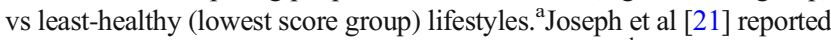
results in African-American, Asian and white ethnicity. ${ }^{\mathrm{b}}$ Some studies did not report the number of participants and cases in stratified analyses. ${ }^{c} \mathrm{Li}$ et al [23] and Liu et al [24] reported results of stratified analyses according to age groups. ${ }^{\mathrm{d}}$ Effoe et al [18] investigated the association between LS7 and risk of incident type 2 diabetes in the Jackson Heart Study, whereas the lifestyle score presented in Joseph et al [22] gave more weight to sleeping. Thus, Effoe et al [18] was used in the stratified analysis. ${ }^{e}$ Five

large study involving 207,479 participants from the USA found that the associations between combined lifestyle factors and incident type 2 diabetes were consistent among normalweight, overweight and obese individuals [11] and in participants with and without a family history of diabetes. However, another study conducted in 3252 African-Americans found that the association was stronger in non-obese participants and normoglycaemic participants, compared with obese commonly used factors, including alcohol drinking, body weight, diet, physical activity and smoking, were considered. However, all studies included physical activity in scores, and only Nguyen et al [28] did not include diet or smoking, and so we have not shown a 'Diet and smoking excluded' category. Joseph et al [22] did not include alcohol drinking or BMI. The Li et al [23] and Shan et al [29] studies were conducted in the Nurses' Health Study and Nurses' Health Study II; however, the Li et al [23] study included all five factors, whereas the Shan et al [29] study did not include alcohol drinking. Thus, these two studies were both included in this stratified analysis in the 'All five factors' and 'Alcohol drinking excluded' categories, respectively. NA, not available

participants and those with impaired fasting glucose/elevated $\mathrm{HbA}_{1 \mathrm{c}}(5.7-6.4 \%)$, respectively, although the sample size was relatively small [22]. Hence, more evidence is needed to answer the question of whether the associations between combined lifestyle factors and incident type 2 diabetes are equivalent in high-risk and low-risk populations; this could facilitate decisions made about what is pivotal for interventions in different populations. 
Fig. 4 Associations of combined lifestyle factors with mortality risk and incident CVD among diabetic individuals. The forest plot shows the HRs (circles) and 95\% CIs comparing people with the healthiest (highest score group) vs least-healthy (lowest score group) lifestyles for mortality and CVD risk in diabetic individuals. The diamond represents the pooled HR. ${ }^{\mathrm{a}}$ The OR was reported in the study and was transformed into RR, which was then used in the pooled analysis. ${ }^{\mathrm{b}}$ The study used mortality data up to the end of 2009. We accessed the data and updated the analysis using mortality data up to the end of 2016

\begin{tabular}{|c|c|c|c|}
\hline Study & Comparison & $\mathrm{HR}(95 \% \mathrm{Cl})$ & Weight, \% \\
\hline \multicolumn{4}{|l|}{ All-cause mortality } \\
\hline Bonaccio et al (2019) [31] & $3-4$ vs $0-1$ points & $0.62(0.46,0.85)$ & 17.69 \\
\hline Dunkler et al (2016) [32] a & $3.5-5$ vs $0-1$ points & $0.39(0.30,0.51)$ & 18.56 \\
\hline Lin et al (2012) [33] & 4 vs $0-1$ points & $0.29(0.17,0.49)$ & 13.03 \\
\hline Mancini et al (2019) [36] & $6-7$ vs $0-1$ points & $0.13(0.05,0.40)$ & 6.15 \\
\hline Nöthlings et al (2010) [37] & $3-5$ vs 0 points & $0.37(0.20,0.69)$ & 11.41 \\
\hline Odegaard et al (2011) [38] ${ }^{\mathrm{b}}$ & $4-5$ vs 0 points & $0.70(0.53,0.91)$ & 18.43 \\
\hline Patel et al (2018) [39] & $4-5$ vs $0-1$ points & $0.56(0.36,0.88)$ & 14.73 \\
\hline Overall $\left({ }^{2}=74.1 \%, p=0.001\right)$ & Highest vs lowest & $0.44(0.33,0.60)$ & 100.00 \\
\hline \multicolumn{4}{|l|}{ CVD mortality } \\
\hline Bonaccio et al (2019) [31] & $3-4$ vs $0-1$ points & $0.70(0.42,1.16)$ & 28.32 \\
\hline Lin et al (2012) [33] & 4 vs $0-1$ points & $0.24(0.07,0.83)$ & 12.11 \\
\hline Liu et al (2018) [34] & $3-5$ vs 0 points & $0.32(0.20,0.52)$ & 29.19 \\
\hline Odegaard et al (2011) [38] ${ }^{\mathrm{b}}$ & $4-5$ vs 0 points & $0.79(0.51,1.22)$ & 30.39 \\
\hline Overall $(R=70.5 \%, p=0.017)$ & Highest vs lowest & $0.51(0.30,0.86)$ & 100.00 \\
\hline \multicolumn{4}{|l|}{ Cancer mortality } \\
\hline Bonaccio et al (2019) [31] & $3-4$ vs $0-1$ points & $0.66(0.39,1.10)$ & 51.70 \\
\hline Lin et al (2012) [33] & 4 vs $0-1$ points & $0.76(0.23,2.56)$ & 9.45 \\
\hline Odegaard et al (2011) [38] ${ }^{\mathrm{b}}$ & $4-5$ vs 0 points & $0.71(0.39,1.29)$ & 38.85 \\
\hline Overall $(R=0.0 \%, p=0.97)$ & Highest vs lowest & $0.69(0.47,1.00)$ & 100.00 \\
\hline \multicolumn{4}{|l|}{ Incident CVD } \\
\hline Liu et al (2018) [34] & $3-5$ vs 0 points & $0.50(0.38,0.65)$ & 94.80 \\
\hline Long et al (2014) [35] & $3-4$ vs 0 points & $0.24(0.06,0.98)$ & 3.44 \\
\hline Zhang et al (2011) [40] & 5 vs $0-1$ points & $0.27(0.04,2.05)$ & 1.76 \\
\hline Overall $(R=0.0 \%, p=0.51)$ & Highest vs lowest & $0.48(0.37,0.63)$ & 100.00 \\
\hline
\end{tabular}

Our study also raised the important clinical issue of whether a healthy lifestyle also confers significant benefits for the management of type 2 diabetes. We found that compared with diabetic individuals with the least-healthy lifestyle, those with the healthiest lifestyle displayed a 31-56\% lower risk of allcause and cause-specific mortality and 52\% lower risk of incident CVD, supporting the recommendations from WHO [44], ADA [56] and some other organisations [43] that lifestyle modification should be the cornerstone for the management of diabetes. Our results were consistent with the Look AHEAD (Action for Health in Diabetes) trial, a randomised controlled trial conducted in 4734 overweight/obese individuals with type 2 diabetes, in which it was reported that achieving $10 \%$ body weight reduction by a healthy low-energy, lowfat diet and increasing physical activity level during the 4 years of intervention could reduce the risk of primary CVD outcomes by $20 \%$ (HR 0.80 [95\% CI $0.65,0.99$ ]) [57]. In addition, diabetic microvascular complications also need to be considered. Several studies suggest that body weight [58], physical activity [59], diet [60], alcohol drinking [61] and smoking [62] are independently associated with microvascular complications among diabetic individuals. However, no prospective cohort study has investigated the association between combined lifestyle factors and diabetic microvascular complications, thus we could not summarise the evidence.
Previous randomised controlled trials found that intensive lifestyle intervention could reduce the risk of microvascular complications among individuals with impaired glucose tolerance or impaired fasting glucose $[6,63]$. However, considering the aforementioned limitations of randomised controlled trials, large prospective observational studies are urgently warranted for elucidating the associations between combined lifestyle factors and diabetic microvascular complications.

Our study is the first systematic review and meta-analysis to summarise the relationship between combined lifestyle factors and incident type 2 diabetes as well as the risk of mortality and incident CVD among diabetic individuals. We followed the standard procedures of the Meta-analysis of Observational Studies in Epidemiology guideline and included 26 studies with over 1 million participants in the metaanalysis. We had sufficient power to perform many stratified analyses and the results were largely consistent. However, several limitations should also be acknowledged. First, most studies were conducted in high-income countries and participants were mostly of white ethnicity, thus more evidence from other populations is still needed. Second, the definitions and combinations of healthy lifestyle factors varied across studies and this could generate potential heterogeneity. However, the differences among subgroups were not significant. Third, limited studies were available for mortality and incident CVD 
risk in diabetic individuals, which restricted us from conducting further stratified analyses. Last, type 2 diabetes is now increasingly seen in adolescents and young adults [64] and more studies are needed to prospectively investigate the role of combined lifestyle factors in the development of type 2 diabetes in this population.

In conclusion, adopting a healthy lifestyle is associated with a substantially lower risk of type 2 diabetes and risk of mortality and incident CVD among individuals with diabetes. The results were generally consistent among participants from different socioeconomic backgrounds and baseline characteristics. Given that the proportion of individuals with the healthiest lifestyle was low in most populations, promotion of an overall healthy lifestyle, instead of tackling one particular lifestyle factor, should be a public health priority for all countries. At the individual level, people are encouraged to maintain optimal weight, avoid smoking and heavy drinking, adopt a healthy diet and increase physical activity levels. At the population level, governments and organisations should incorporate encouragement of healthy lifestyles into all health-related policies and guidelines and should facilitate the environmental change needed to make healthy lifestyle choices accessible, affordable and sustainable. Our study also suggests that future studies should focus on the associations between combined lifestyle factors and microvascular complications and longterm outcomes among diabetic individuals, to provide important evidence for diabetes management.

Acknowledgements The authors cordially acknowledge Y. Li (Department of Nutrition, Harvard T.H. Chan School of Public Health, USA) for providing additional data and information pertinent to original reports.

Data availability The datasets generated during and/or analysed during the current study are available from the corresponding author on reasonable request.

Funding AP was supported by the National Key Research and Development Program of China (2017YFC0907500 and 2017YFC0907504), National Nature Science Foundation of China (81773517) and Hubei Province Science Fund for Distinguished Young Scholars (2018CFA033). XFP was supported by the China Postdoctoral Science Foundation (176596) and International Postdoctoral Exchange Fellowship of the China Postdoctoral Council (20180062). MH was supported by the National Nature Science Foundation of China (81522040) and the National Key Research and Development Program of China (2017YFC0907501). The study sponsor was not involved in the design of the study, the collection, analysis and interpretation of data, writing the report or the decision to submit the report for publication.

Duality of interest The authors declare that there is no duality of interest associated with this manuscript.

Contribution statement $\mathrm{YbZ}, \mathrm{XFP}$ and AP designed the research. YbZ and JC did the literature search. YbZ, JC, LX, AC, YgZ, JW and HL reviewed studies for inclusion and performed data extraction and checking. YbZ and JC performed meta-analyses. YbZ, KY, KG, MH and $\mathrm{AP}$ contributed to the interpretation of data. $\mathrm{YbZ}$ drafted the article. XFP, JC, LX, AC, YgZ, JW, HL, KY, KG, MH and AP contributed to the critical revision of the manuscript for important intellectual content. All authors approved the final manuscript. AP is the guarantor of this work.

\section{References}

1. James SL, Abate D, Abate KH et al (2018) Global, regional, and national incidence, prevalence, and years lived with disability for 354 diseases and injuries for 195 countries and territories, 19902017: a systematic analysis for the Global Burden of Disease Study 2017. Lancet 392(10159):1789-1858. https://doi.org/10.1016/ S0140-6736(18)32279-7

2. Chatterjee S, Khunti K, Davies MJ (2017) Type 2 diabetes. Lancet 389(10085):2239-2251. https://doi.org/10.1016/S0140-6736(17) 30058-2

3. Zheng Y, Ley SH, Hu FB (2018) Global aetiology and epidemiology of type 2 diabetes mellitus and its complications. Nat Rev Endocrinol 14(2):88-98. https://doi.org/10.1038/nrendo.2017.151

4. Bennett JE, Stevens GA, Mathers CD et al (2018) NCD Countdown 2030: worldwide trends in non-communicable disease mortality and progress towards Sustainable Development Goal target 3.4. Lancet 392(10152):1072-1088. https://doi.org/10.1016/ S0140-6736(18)31992-5

5. World Health Organization (2017) 'Best buys' and other recommended interventions for the prevention and control of noncommunicable diseases. Available from http://www.who.int/ iris/handle/10665/259232. Accessed 5 May 2019

6. Gong Q, Zhang P, Wang J et al (2019) Morbidity and mortality after lifestyle intervention for people with impaired glucose tolerance: 30-year results of the Da Qing Diabetes Prevention Outcome Study. Lancet Diabetes Endocrinol 7(6):452-461. https://doi.org/ 10.1016/S2213-8587(19)30093-2

7. Haw JS, Galaviz KI, Straus AN et al (2017) Long-term sustainability of diabetes prevention approaches: a systematic review and meta-analysis of randomized clinical trials. JAMA Intern Med 177(12):1808-1817. https://doi.org/10.1001/jamainternmed.2017. 6040

8. Knowler WC, Barrett-Connor E, Fowler SE et al (2002) Reduction in the incidence of type 2 diabetes with lifestyle intervention or metformin. N Engl J Med 346(6):393-403

9. Lindstrom J, Ilanne-Parikka P, Peltonen M et al (2006) Sustained reduction in the incidence of type 2 diabetes by lifestyle intervention: follow-up of the Finnish Diabetes Prevention Study. Lancet 368(9548):1673-1679. https://doi.org/10.1016/S0140-6736(06) 69701-8

10. Stroup DF, Berlin JA, Morton SC et al (2000) Meta-analysis of observational studies in epidemiology: a proposal for reporting. Meta-analysis Of Observational Studies in Epidemiology (MOOSE) group. JAMA 283(15):2008-2012. https://doi.org/10. 1001/jama.283.15.2008

11. Reis JP, Loria CM, Sorlie PD, Park Y, Hollenbeck A, Schatzkin A (2011) Lifestyle factors and risk for new-onset diabetes a population-based cohort study. Ann Intern Med 155(5):292-299. https://doi.org/10.7326/0003-4819-155-5-201109060-00006

12. Fretts AM, Howard BV, McKnight B et al (2014) Life s Simple 7 and incidence of diabetes among American Indians: the Strong Heart Family Study. Diabetes Care 37(8):2240-2245. https://doi. org/10.2337/dc13-2267

13. Lloyd-Jones DM, Hong Y, Labarthe D et al (2010) Defining and setting national goals for cardiovascular health promotion and disease reduction: the American Heart Association s strategic Impact Goal through 2020 and beyond. Circulation 121(4):586-613. https://doi.org/10.1161/CIRCULATIONAHA.109.192703 
14. Cook DA, Reed DA (2015) Appraising the quality of medical education research methods: the Medical Education Research Study Quality Instrument and the Newcastle-Ottawa Scale-Education. Acad Med 90(8):1067-1076. https://doi.org/10.1097/ACM. 0000000000000786

15. Higgins JPT, Green S (2006) Cochrane handbook for systematic reviews of interventions. John Wiley, Chichester

16. Borenstein M, Hedges LV, Higgins JPT, Rothstein HR (2009) Introduction to meta-analysis. John Wiley, Chichester. https://doi. org/10.1002/9780470743386

17. Dow C, Balkau B, Bonnet F et al (2019) Strong adherence to dietary and lifestyle recommendations is associated with decreased type 2 diabetes risk in the AusDiab cohort study. Prev Med 123: 208-216. https://doi.org/10.1016/j.ypmed.2019.03.006

18. Effoe VS, Carnethon MR, Echouffo-Tcheugui JB et al (2017) The American Heart Association ideal cardiovascular health and incident type 2 diabetes mellitus among blacks: the Jackson Heart Study. J Am Heart Assoc 6(6):e005008

19. Ford ES, Bergmann MM, Kroger J, Schienkiewitz A, Weikert C, Boeing H (2009) Healthy living is the best revenge: findings from the European Prospective Investigation Into Cancer and NutritionPotsdam study. Arch Intern Med 169(15):1355-1362. https://doi. org/10.1001/archinternmed.2009.237

20. Joosten MM, Grobbee DE, van der AD, Verschuren WM, Hendriks HF, Beulens JW (2010) Combined effect of alcohol consumption and lifestyle behaviors on risk of type 2 diabetes. Am J Clin Nutr 91(6):1777-1783. https://doi.org/10.3945/ajcn.2010.29170

21. Joseph JJ, Echouffo-Tcheugui JB, Carnethon MR et al (2016) The association of ideal cardiovascular health with incident type 2 diabetes mellitus: the Multi-Ethnic Study of Atherosclerosis. Diabetologia 59(9):1893-1903. https://doi.org/10.1007/s00125016-4003-7

22. Joseph JJ, Echouffo-Tcheugui JB, Talegawkar SA et al (2017) Modifiable lifestyle risk factors and incident diabetes in African Americans. Am J Prev Med 53(5):e165-e174. https://doi.org/10. 1016/j.amepre.2017.06.018

23. Li Y, Ley SH, Tobias DK et al (2015) Birth weight and later life adherence to unhealthy lifestyles in predicting type 2 diabetes: prospective cohort study. BMJ 351:h3672

24. Liu X, Cui L, Wang A et al (2016) Cumulative exposure to ideal cardiovascular health and incident diabetes in a Chinese population: the Kailuan Study. J Am Heart Assoc 5(9):e004132

25. Long GH, Johansson I, Rolandsson O et al (2015) Healthy behaviours and 10-year incidence of diabetes: a population cohort study. Prev Med 71:121-127. https://doi.org/10.1016/j.ypmed.2014.12. 013

26. Lv J, Yu C, Guo Y et al (2017) Adherence to a healthy lifestyle and the risk of type 2 diabetes in Chinese adults. Int J Epidemiol 46(5): 1410-1420. https://doi.org/10.1093/ije/dyx074

27. Mozaffarian D, Kamineni A, Carnethon M, Djousse L, Mukamal KJ, Siscovick D (2009) Lifestyle risk factors and new-onset diabetes mellitus in older adults: the Cardiovascular Health study. Arch Intern Med 169(8):798-807. https://doi.org/10.1001/ archinternmed.2009.21

28. Nguyen B, Bauman A, Ding D (2017) Incident type 2 diabetes in a large Australian cohort study: does physical activity or sitting time alter the risk associated with body mass index? J Phys Act Health 14(1):13-19. https://doi.org/10.1123/jpah.2016-0184

29. Shan Z, Li Y, Zong G et al (2018) Rotating night shift work and adherence to unhealthy lifestyle in predicting risk of type 2 diabetes: results from two large US cohorts of female nurses. BMJ 363: $\mathrm{k} 4641$

30. Tatsumi Y, Ohno Y, Morimoto A, Nishigaki Y, Mizuno S, Watanabe S (2013) Lifestyle and the risk of diabetes mellitus in a Japanese population. J Behav Med 36(3):225-233. https://doi.org/10.1007/ s10865-012-9427-z
31. Bonaccio M, Di Castelnuovo A, Costanzo S et al (2019) Impact of combined healthy lifestyle factors on survival in an adult general population and in high-risk groups: prospective results from the Moli-sani Study. J Intern Med 286(2):207-220. https://doi.org/10. 1111/joim. 12907

32. Dunkler D, Kohl M, Teo KK et al (2016) Population-attributable fractions of modifiable lifestyle factors for CKD and mortality in individuals with type 2 diabetes: a cohort study. Am J Kidney Dis 68(1):29-40. https://doi.org/10.1053/j.ajkd.2015.12.019

33. Lin CC, Li CI, Liu CS et al (2012) Impact of lifestyle-related factors on all-cause and cause-specific mortality in patients with type 2 diabetes: the Taichung Diabetes Study. Diabetes Care 35(1):105112. https://doi.org/10.2337/dc11-0930

34. Liu G, Li Y, Hu Y et al (2018) Influence of lifestyle on incident cardiovascular disease and mortality in patients with diabetes mellitus. J Am Coll Cardiol 71(25):2867-2876. https://doi.org/10. 1016/j.jacc.2018.04.027

35. Long GH, Cooper AJ, Wareham NJ, Griffin SJ, Simmons RK (2014) Healthy behavior change and cardiovascular outcomes in newly diagnosed type 2 diabetic patients: a cohort analysis of the ADDITION-Cambridge study. Diabetes Care 37(6):1712-1720. https://doi.org/10.2337/dc13-1731

36. Mancini GBJ, Maron DJ, Hartigan PM et al (2019) Lifestyle, glycosylated hemoglobin A1c, and survival among patients with stable ischemic heart disease and diabetes. J Am Coll Cardiol 73(16): 2049-2058. https://doi.org/10.1016/j.jacc.2018.11.067

37. Nöthlings U, Ford ES, Kroger J, Boeing H (2010) Lifestyle factors and mortality among adults with diabetes: findings from the European Prospective Investigation into Cancer and NutritionPotsdam study. J Diabetes 2(2):112-117. https://oi.org/10.1111/j. 1753-0407.2010.00069.x

38. Odegaard AO, Koh WP, Gross MD, Yuan JM, Pereira MA (2011) Combined lifestyle factors and cardiovascular disease mortality in Chinese men and women: the Singapore Chinese health study. Circulation 124(25):2847-2854. https://doi.org/10.1161/ CIRCULATIONAHA.111.048843

39. Patel YR, Gadiraju TV, Gaziano JM, Djousse L (2018) Adherence to healthy lifestyle factors and risk of death in men with diabetes mellitus: the Physicians Health Study. Clin Nutr 37(1):139-143. https://doi.org/10.1016/j.clnu.2016.11.003

40. Zhang Y, Tuomilehto J, Jousilahti P, Wang Y, Antikainen R, Hu G (2011) Lifestyle factors on the risks of ischemic and hemorrhagic stroke. Arch Intern Med 171(20):1811-1818. https://doi.org/10. 1001/archinternmed.2011.443

41. Li G, Zhang P, Wang J et al (2008) The long-term effect of lifestyle interventions to prevent diabetes in the China Da Qing Diabetes Prevention Study: a 20-year follow-up study. Lancet 371(9626): 1783-1789. https://doi.org/10.1016/S0140-6736(08)60766-7

42. American Diabetes Association (2019) 3. Prevention or delay of type 2 diabetes: standards of medical care in diabetes-2019. Diabetes Care 42(Suppl 1):S29-S33. https://doi.org/10.2337/ dc19-S003

43. International Diabetes Federation (2017) IDF Diabetes Atlas, 8th edn. International Diabetes Federation, Brussels

44. World Health Organization (2016) Global report on diabetes. Available from https://apps.who.int/iris/bitstream/handle/10665/ 204871/9789241565257_eng.pdf?sequence=1. Accessed 28 April 2019

45. Aune D, Norat T, Leitzmann M, Tonstad S, Vatten LJ (2015) Physical activity and the risk of type 2 diabetes: a systematic review and dose-response meta-analysis. Eur J Epidemiol 30(7):529-542. https://doi.org/10.1007/s10654-015-0056-Z

46. Jannasch F, Kröger J, Schulze MB (2017) Dietary patterns and type 2 diabetes: a systematic literature review and meta-analysis of prospective studies. J Nutr 147(6):1174-1182. https://doi.org/10.3945/ jn. 116.242552 
47. Pan A, Wang Y, Talaei M, Hu FB, Wu T (2015) Relation of active, passive, and quitting smoking with incident diabetes: a metaanalysis and systematic review. Lancet Diabetes Endocrinol 3(12): 958-967. https://doi.org/10.1016/S2213-8587(15)00316-2

48. Knott C, Bell S, Britton A (2015) Alcohol consumption and the risk of type 2 diabetes: a systematic review and dose-response metaanalysis of more than 1.9 million individuals from 38 observational studies. Diabetes Care 38(9):1804-1812. https://doi.org/10.2337/ dc15-0710

49. Cloostermans L, Wendelvos W, Doornbos G et al (2015) Independent and combined effects of physical activity and body mass index on the development of type 2 diabetes - a meta-analysis of 9 prospective cohort studies. Int J Behav Nutr Phys Act 12(1): 147. https://doi.org/10.1186/s12966-015-0304-3

50. Blüher M (2019) Obesity: global epidemiology and pathogenesis. Nat Rev Endocrinol 15(5):288-298. https://doi.org/10.1038/ s41574-019-0176-8

51. de Walque D (2010) Education, information, and smoking decisions: evidence from smoking histories in the United States, 1940-2000. J Hum Resour 45(3):682-717

52. Carlson A, Frazao E (2014) Food costs, diet quality and energy balance in the United States. Physiol Behav 134:20-31. https:// doi.org/10.1016/j.physbeh.2014.03.001

53. Bommer C, Heesemann E, Sagalova V et al (2017) The global economic burden of diabetes in adults aged 20-79 years: a costof-illness study. Lancet Diabetes Endocrinol 5(6):423-430. https:// doi.org/10.1016/S2213-8587(17)30097-9

54. Chan JCN, Gregg EW, Sargent J, Horton R (2016) Reducing global diabetes burden by implementing solutions and identifying gaps: a Lancet Commission. Lancet 387(10027):1494-1495. https://doi. org/10.1016/S0140-6736(16)30165-9

55. World Health Organization (2017) Noncommunicable diseases progress monitor, 2017. Available from https://www.who.int/nmh/ publications/ncd-progress-monitor-2017/en/. Accessed 5 May 2019

56. American Diabetes Association (2019) 5. Lifestyle management: standards of medical care in diabetes-2019. Diabetes Care 42(Suppl 1):S46-S60. https://doi.org/10.2337/dc19-S005
57. Gregg EW, Jakicic JM, Blackburn G et al (2016) Association of the magnitude of weight loss and changes in physical fitness with longterm cardiovascular disease outcomes in overweight or obese people with type 2 diabetes: a post-hoc analysis of the Look AHEAD randomised clinical trial. Lancet Diabetes Endocrinol 4(11):913921

58. Sjostrom L, Peltonen M, Jacobson P et al (2014) Association of bariatric surgery with long-term remission of type 2 diabetes and with microvascular and macrovascular complications. JAMA 311(22):2297-2304. https://doi.org/10.1001/jama.2014.5988

59. Blomster JI, Chow CK, Zoungas S et al (2013) The influence of physical activity on vascular complications and mortality in patients with type 2 diabetes mellitus. Diabetes Obes Metab 15(11):10081012. https://doi.org/10.1111/dom.12122

60. Diaz-Lopez A, Babio N, Martinez-Gonzalez MA et al (2015) Mediterranean diet, retinopathy, nephropathy, and microvascular diabetes complications: a post hoc analysis of a randomized trial. Diabetes Care 38(11):2134-2141. https://doi.org/10.2337/dc151117

61. Blomster JI, Zoungas S, Chalmers J et al (2014) The relationship between alcohol consumption and vascular complications and mortality in individuals with type 2 diabetes. Diabetes Care 37(5): 1353-1359. https://doi.org/10.2337/dc13-2727

62. Zhu P, Pan X-F, Sheng L, Chen H, Pan A (2017) Cigarette smoking, diabetes, and diabetes complications: call for urgent action. Curr Diab Rep 17(9):78

63. Diabetes Prevention Program Research Group (2015) Long-term effects of lifestyle intervention or metformin on diabetes development and microvascular complications over 15-year follow-up: the Diabetes Prevention Program Outcomes Study. Lancet Diabetes Endocrinol 3(11):866-875

64. Lascar N, Brown J, Pattison H, Barnett AH, Bailey CJ, Bellary S (2018) Type 2 diabetes in adolescents and young adults. Lancet Diabetes Endocrinol 6(1):69-80. https://doi.org/10.1016/S22138587(17)30186-9

Publisher's note Springer Nature remains neutral with regard to jurisdictional claims in published maps and institutional affiliations. 